\title{
Cerebral Venous Sinus Thrombosis Associated with Immune Thrombocytopenic Purpura: A Case Report
}

\author{
Karen DV. Hernandez, Ma. Angelina L. Mirasol and Maria Cristina Z. San Jose
}

Department of Neurosciences, College of Medicine and Philippine General Hospital, University of the Philippines Manila

\begin{abstract}
Cerebral venous sinus thrombosis (CVST) constitutes a rare but serious stroke mechanism associated with hypercoaguable states. Immune thrombocytopenia (ITP) is a common hematologic disorder that results in an increased bleeding risk. CVST on the background of thrombocytopenia could be considered a clinical paradox. The co-existence of hyper and hypocoagulable states create management dilemmas. We present the case of an 18-year-old male hospitalized for sudden severe headache and seizures. Thrombosis of the sagittal sinus was confirmed with angiography. Work up of the persistently low platelet count revealed ITP. He was given medical decompression and steroids, with note of subsequent improvement in his functional status and platelet count.
\end{abstract}

Key Words: venous thrombosis, thrombocytopenia, headache

\section{Introduction}

The rare occurrence of sagittal sinus thrombosis associated with thrombocytopenia has been reported only in two very young patients, 22 and 42 months old, with the former having a predilection to thrombosis due to presence of lupus antibody and the latter having no identified thrombophilic risk factor. ${ }^{1}$ In cases of immune thrombocytopenia, the destruction of platelets by antibodies results in an increased bleeding risk rather than a prothrombic state. The occurrence of a significant thrombotic phenomenon on the background of immune thrombocytopenia can be considered a clinical paradox.

This report aims to present in detail a clinical paradox, an unusual association of diseases leading to various dilemmas in management and an unusual setting of care. We aim to present and address important issues in the diagnosis and management of such a case. We aim to add

Paper presented at the Philippine Neurological Association Annual Research Forum, November 2011, Edsa Shangri-la Hotel, Mandaluyong City (1st place - Case Report category).

Poster presented at the Asia Pacific Stroke Conference, September 2012, Tokyo, Japan.

Corresponding author: Karen DV. Hernandez, MD

Department of Neurosciences

Philippine General Hospital

University of the Philippines Manila

Taft Avenue, Ermita, Manila 1000 Philippines

Telefax No.: +632 5548462

Email: olren0017@yahoo.com evidence to the scarce literature about this rare phenomenon, including the dilemmas encountered and some plausible mechanisms for the occurrence of thrombotic events in patients with low platelet count.

\section{Case Presentation}

An 18-year-old college senior was playing basketball when he was accidentally hit by the ball on his left eye. He experienced sudden severe dizziness but no immediate deficits were observed. For several days, he experienced persistent pulsating bitemporal headache associated with vomiting, prompting hospital admission. He had an episode of generalized tonic clonic seizure, after which he developed right-sided hemiparesis and dysarthria. Initial cranial computed tomography (CT) scan revealed a hyperdensity in the left cerebral cortical sulci and cisterns (Figure 1), suggestive of subarachnoid hemorrhage. CT angiogram revealed an intraparenchymal hematoma in the left parietal parasagittal lobe near the vertex and hypodense filling defects in the superior sagittal sinus suggestive of venous thrombosis (Figure 2). He was subsequently transferred to our institution for further management.

On examination, he was drowsy and dysarthric, occasionally oriented to person and place. Papilledema and subhyaloid hemorrhage were noted over the right eye. He had right hemianopia, central facial palsy, hemiparesis, and hemianesthesia. Repeat cranial CT, performed 1 week after the initial scan, revealed bilateral hemorrhages in the parieto-occipital areas consistent with the worsening of the neurologic deficits. Four-vessel cerebral angiogram showed delayed filling of the fronto-parietal cortical veins and the superior sagittal sinus during the venous phase (Figure 3). All tests for hypercoagulable disorders were normal (Table 1). He was also negative for anti-nuclear antibody (ANA).

Table 1. Summary of test results for hypercoagulability disorders

\begin{tabular}{lcc}
\hline & Results & Reference Range \\
\hline KCT & 57 & $50-90$ \\
PTT & 45 & $25-40$ \\
DRWT & 39 & $31-44$ \\
Antithrombin & 439 & Male: $260-378$ \\
Protein C & $76.2 \%$ & $70-140 \%$ \\
\hline KCT: Kaoli &
\end{tabular}

KCT: Kaolin clotting time, PTT: Prothrombin time; DRVVT: Dilute Ruddell's Viper Venom time 


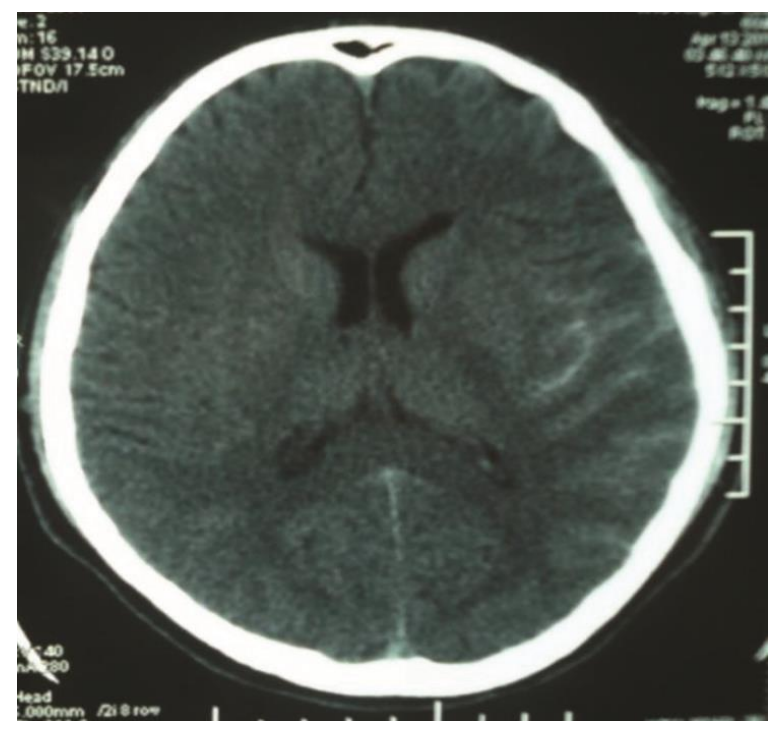

Figure 1. Plain cranial CT scan showed increased density of the left cerebral cortical sulci and cisterns. Cerebral aneurysm vs vascular malformation was initially considered.

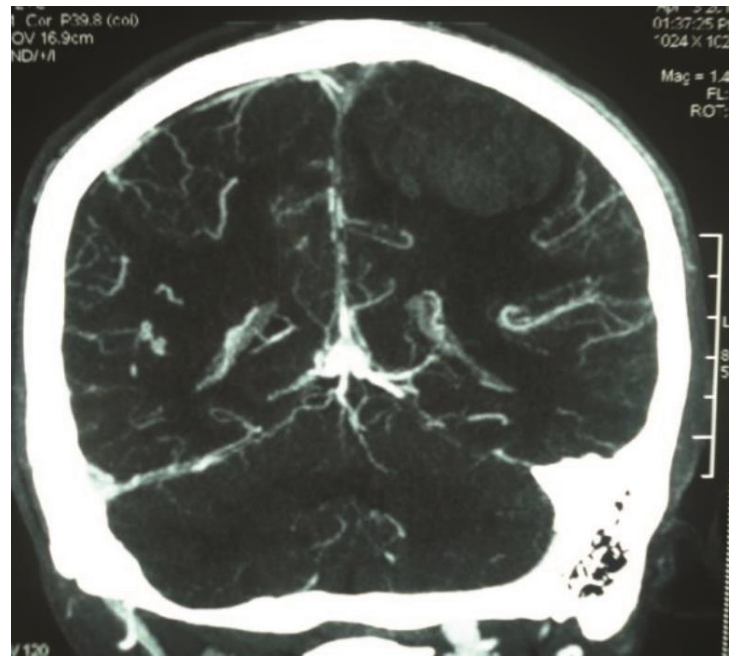

Figure 2. CT angiogram showed intraparenchymal hematoma on the L parietal prasagittal lobe $3 \times 4.7 \times 3 \mathrm{~cm}$. The classic empty delta sign is noted (arrow), characterized by the presence of thrombus (hypodensity) within the superior sagittal sinus, surrounded by a triangular area of enhancement, indicative of sinus thrombosis and present in $35 \%$ of published cases. ${ }^{2}$

An incidental finding of persistently low platelet count $(33,000 / \mu \mathrm{L})$ was noted; the rest of the complete blood count (CBC) were within normal range except for mild leukocytosis. Dengue rapid tests yielded negative results. Ultrasonographic assessment of the liver, spleen, and kidneys revealed normal organs. Subsequent CBCs revealed persistently decreasing platelet counts, initially posing a dilemma in the further work up for sagittal sinus thrombosis, which involves a 4-vessel angiogram, a more invasive procedure that poses greater risks for bleeding. $\mathrm{He}$ was transfused with 6 units of platelet concentrate 2 hours prior and another 4 units immediately after the angiogram.

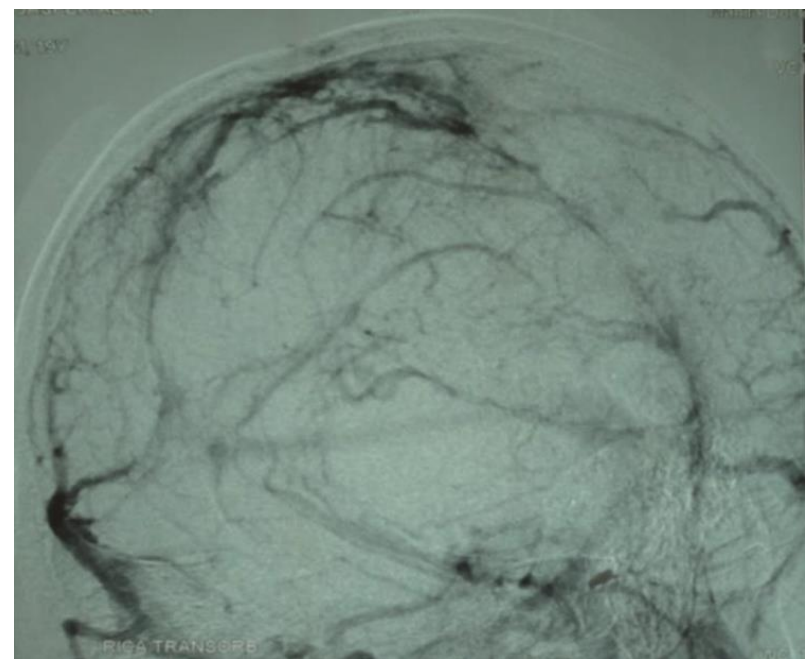

Figure 3. 4-vessel angiogram showed delayed filling of the fronto-parietal cortical veis and superior sagittal sinus with incomplete, irregular and poor opacification of the midsegment of the posterior segment of the superior sagittal sinus. These findings are consistent with partial thrombosis of the superior sagittal sinus.

Further work up included a peripheral blood smear (Figure 4) and bone marrow aspirate biopsy which showed normocellular marrow with mild to moderate megakaryocytic hyperplasia (Figure 5), indicative of immune/idiopathic thrombocytopenia (ITP).

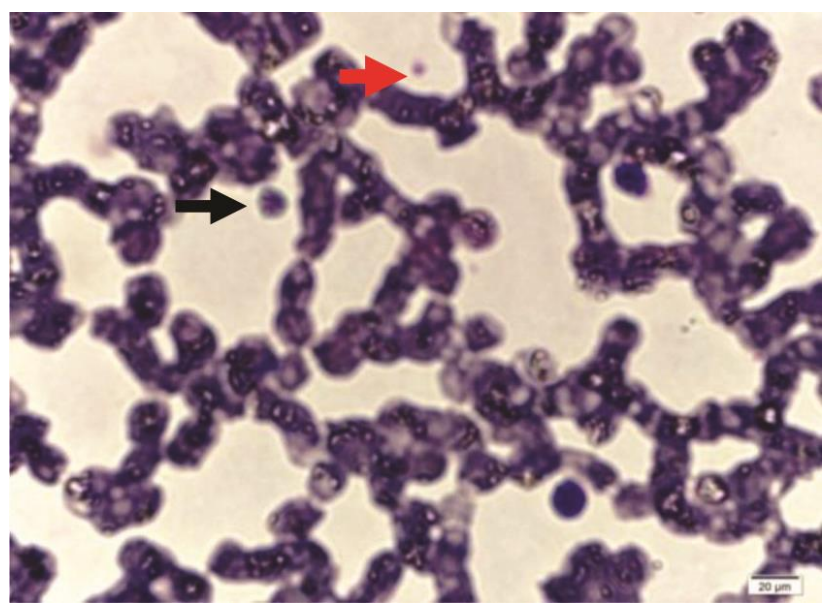

Figure 4. Peripheral blood smear showed decreased number of platelets (red arrow). Normal range of platelet per oil immersion field is 7-21. ${ }^{3}$ The notable increase in size of platelets (black arrow) is characteristic of ITP. 


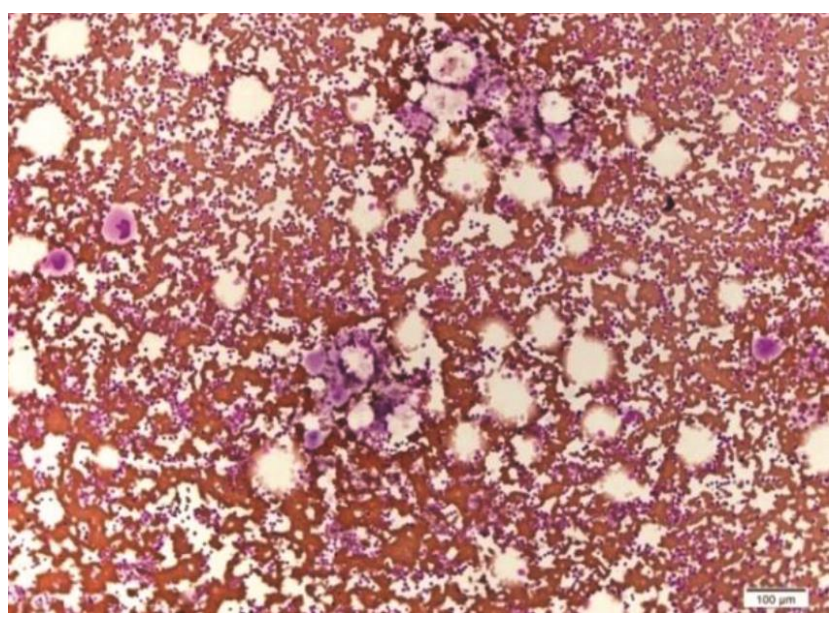

Figure 5. Bone marrow aspiration biopsy showed increased proportion of immature megakaryocytes secondary to the accelerated platelet destruction that leads to the stimulation of megakaryocytopoiesis. ${ }^{4}$

Our patient was started on mannitol, omeprazole, levetiracetam, citicoline, and dexamethasone. The dexamethasone was later shifted to prednisone. Though the ideal management for sagittal sinus thrombosis includes anticoagulation ${ }^{5}$, the presence of a low platelet count posed a clinical dilemma. Endovascular coiling was a suitable alternative; however, the procedure's cost was the main limiting factor. Steroid treatment was started to address the ITP while closely monitoring the patient's neurologic status.

In the next few weeks, improvement in the patient's sensorium was noted, with gradual improvement of neurologic deficits including right hemiplegia. He was subsequently referred to rehabilitation medicine for early mobilization exercises and prevention of complications of prolonged immobilization. He was discharged on the 34th hospital day with residual weakness of the right extremities, and was maintained on low dose prednisone. On follow up after a month, he was ambulatory without need of assistance. Markedly improved upper extremity strength was observed, with resolution of the sensory deficits, hemineglect, and homonymous hemianopia. He was independent in most aspects of activities of daily living and was able to go back to school. His platelet count continued to improve after discharge and the steroid dose was gradually tapered and eventually discontinued. Repeat imaging studies using magnetic resonance venogram (MRV) performed 5 months after the ictus showed resolution of the veno-occlusive disease and patency of the superior sagittal sinus (Figure 6).

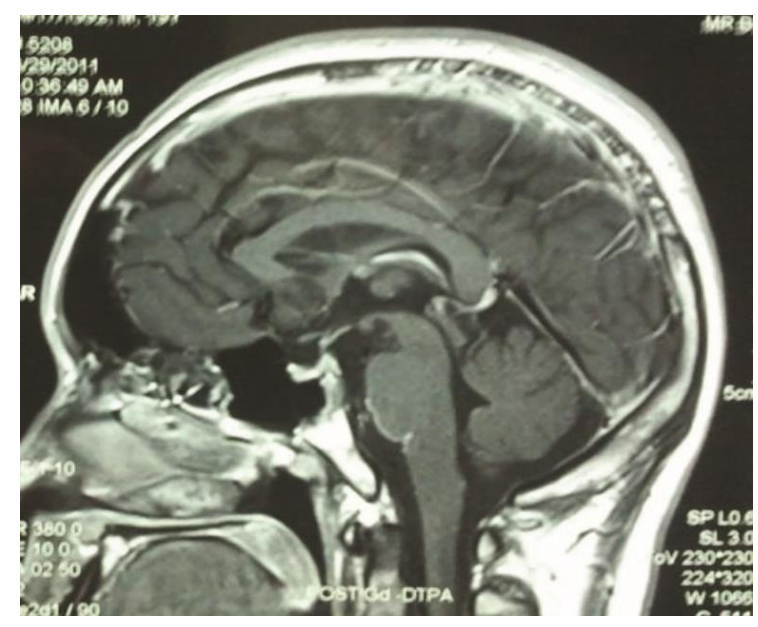

Figure 6. Cranial MRI/MRV done five months post ictus showed patency of the superior sagittal sinus, correlating to the marked clinical improvement.

\section{Discussion}

Cerebral venous sinus thrombosis (CVST) is an uncommon type of stroke mechanism, accounting for $<1 \%$ of all strokes, with a higher incidence among females. ${ }^{5}$ Though progressive headache is the most frequent symptom, the clinical spectrum is protean, and includes focal deficits and seizures. The main sinuses affected are the superior sagittal and the lateral sinuses. In the former, venous infarcts are typically located near the midline, in the rolandic and parieto-occipital regions, similar in our patient's case. The obstruction results in increased venous pressure leading to disruption of the blood-brain barrier, and venous hemorrhage. In rare cases, patients with CVST may present as cortical subarachnoid hemorrhage on imaging, with the blood usually along the cortical convexity or sylvian cistern, with notable sparing of the basal cisterns. ${ }^{6}$ This is consistent with our patient's initial cranial CT.

The disturbed equilibrium of prothrombic and thrombolytic processes is considered the main underlying pathology. Hereditary prothrombotic conditions such as Factor V Leiden, deficiency of proteins $\mathrm{C}$ and $\mathrm{S}$ and antithrombin III as well as prothrombin gene mutations account for $10-15 \%$ of cases. ${ }^{7}$

Idiopathic or immune thrombocytopenic purpura (ITP) is an acquired, immune-mediated disorder characterized by isolated thrombocytopenia (defined as a peripheral blood platelet count less than $100 \times 10^{9} / \mathrm{L}$ ) and the absence of any apparent initiating and/or underlying cause. ${ }^{8}$ Most recently, it is regarded as immune thrombocytopenia to give emphasis to the immunologic destruction of otherwise normal platelets in response to an unknown stimulus. ${ }^{9}$ Corticosteroid is the standard initial treatment.

The clinical paradox associating thrombocytopenia with a prothrombic state has been highlighted in some recent studies. In a restrospective cohort study by Sarpatwari et al., 
adults with primary immune thrombocytopenia were found to be at risk of thromboembolic events. After over a median follow-up period of 4 years, adjusted hazard ratios of 1.58 (95\% CI, 1.01-2.48), 1.37 (95\% CI, 0.94-2.00), and 1.41 (95\% CI, 1.04-1.91) were found for venous, arterial, and combined (arterial and venous) thromboembolic events, respectively, when comparing the ITP cohort with the disease free cohort. $^{10}$ Some plausible mechanisms include increased platelet microparticle thrombogenicity and aggregation following peripheral destruction and the activity of antiphospholipid antibodies. ${ }^{10}$ However, further work is needed to confirm the uncovered association.

In such a setting where there seems to be a coexistence of both hypo and hypercoaguable states, dilemmas in management are inevitable. The persistent decrease in platelet count initially posed limitations as the patient needed to undergo an invasive diagnostic procedure. Even the use of steroids for the management of immune thrombocytopenia is controversial in the presence of a thrombotic event, as chronic steroid use potentially augments the hypercoaguable state. ${ }^{11}$ On the other hand, anticoagulation, the ideal management for CVST, is controversial in the setting of severely decreased platelet. Facing multiple dilemmas, careful coordination between all the physicians involved in the care of the patient is essential. There is a need to evaluate all procedures to be performed especially if these entail bleeding risks or could potentially worsen the thrombotic event. Analysis of the benefits against the potential risks takes utmost priority.

\section{Conclusion}

This report details a case of sagittal sinus thrombosis in an 18-year-old male with concomitant immune thrombocytopenic purpura. The paradoxical thrombotic event is attributed to the increased platelet microparticle thrombogenicity after immune platelet destruction. In such cases where the patient is at risk for both thrombotic events and bleeding diathesis, careful coordination between the specialists must be ensured to create a balance between all potential hypocoaguable and hypercoaguable factors.

\section{References}

1. Alper G, Berrak SG, Ekinci G, Canpolat C, Erzen C. Sagittal sinus thrombosis associated with thrombocytopenia: a report of two patients. Pediatr Neurol. 1999; 21(2):573-5.

2. Lee E. The empty delta sign. Radiology. 2002; 224(3):788-9.

3. Tien SL. Validating the Platelet Count. Singapore Med J. 1995; 36(3):2556.

4. Podolak-Dawidziak M. Megakaryocyte progenitors in immune thrombocytopenic purpura (ITP). Thromb Res. 1991; 62(1-2):93-6.

5. Allroggen $\mathrm{H}$, Abbott RJ. Cerebral venous sinus thrombosis. Postgrad Med J. 2000; 76(891):12-5.

6. Oppenheim C, Domingo V, Gauvrit, JY, et al. Subarachnoid hemorrhage as the initial presentation of dural sinus thrombosis. AJNR Am J Neuroradiol. 2005;26(3):614-7.

7. Deschiens MA, Conard J, Horellou, MH, et al. Coagulation studies, factor V Leiden, and anticardiolipin antibodies in 40 cases of cerebral venous thrombosis. Stroke. 1996; 27(10):1724-30.

8. McCare K. Immune thrombocytopenia: no longer "idiopathic". Cleve Clin J Med. 2011; 78(6):358-73.

9. Rodeghiero F, Stasi R, Gernsheimer T, et al. Standardization of terminology, definitions and outcome criteria in immune thrombocytopenic purpura of adults and children: report from an international working group. Blood. 2009:113(11):2386-93.

10. Sarpatwari A, Bennett D, Logie, JW, et al. Thromboembolic events among adult patients with primary immune thrombocytopenia in the United Kingdom General Practice Research Database. Haematologica. 2010; 95(7):1167-75.

11. Patrassi GM, Sartori MT, Rigotti P, et al. Reduced fibrinolytic potential one year after kidney transplantation: Relationship to long-term steroid treatment. Transplantation. 1995; 59(10):1416-20. 